\title{
ARTICLE
}

\section{Thermodynamic stability of ligand-protected metal nanoclusters}

\author{
Michael G. Taylor ${ }^{1} \&$ Giannis Mpourmpakis ${ }^{1}$
}

Despite the great advances in synthesis and structural determination of atomically precise, thiolate-protected metal nanoclusters, our understanding of the driving forces for their colloidal stabilization is very limited. Currently there is a lack of models able to describe the thermodynamic stability of these 'magic-number' colloidal nanoclusters as a function of their atomic-level structural characteristics. Herein, we introduce the thermodynamic stability theory, derived from first principles, which is able to address stability of thiolate-protected metal nanoclusters as a function of the number of metal core atoms and thiolates on the nanocluster shell. Surprisingly, we reveal a fine energy balance between the core cohesive energy and the shell-to-core binding energy that appears to drive nanocluster stabilization. Our theory applies to both charged and neutral systems and captures a large number of experimental observations. Importantly, it opens new avenues for accelerating the discovery of stable, atomically precise, colloidal metal nanoclusters.

\footnotetext{
${ }^{1}$ Department of Chemical Engineering, University of Pittsburgh, Pittsburgh, Pennsylvania 15261, USA. Correspondence and requests for materials should be addressed to G.M. (email: gmpourmp@pitt.edu).
} 
M etal nanoclusters (NCs) are an exciting class of materials due to their unique properties that differ from both bulk and atomic-scale behaviour. Colloidal NCs stabilized by the presence of thiolate molecules on their surface, in particular, have broad applications that range from biolabeling to targeted drug delivery to catalysis ${ }^{1-3}$. In the Brust-Schiffrin-type syntheses of these colloidal NCs, metal salts (most notably, $\mathrm{Au}$ ) are reduced in the presence of thiolate ligands to produce NCs of specific sizes depending on the ligands and reaction conditions used ${ }^{4,5}$. The resulting size (and shape) of the NCs, in turn, determines their physicochemical properties ${ }^{2}$. Advances in materials characterization have enabled the crystal structure determination of a series of thermally stable (magic-number) thiolated metal NCs $\left(\mathrm{M}_{n} \mathrm{SR}_{m}\right.$, where $\mathrm{M}=$ metal and $\mathrm{SR}=$ thiolate group) consisting of up to a few hundred atoms $s^{5-19}$. First-principles-based computational modelling has also been employed to probe structural and electronic properties of these magic-number clusters. In particular, the 'divide-and-protect' theory emerged in an effort to rationalize the observed structural characteristics of $\mathrm{Au} \mathrm{NC}$ and the 'superatom' theory to explain the magic-number NC stability $11,14,15,17,20,21$

The divide-and-protect theory suggests that Au NCs form from maximizing $\mathrm{Au}-\mathrm{Au}$ and $\mathrm{Au}-\mathrm{S}$ interactions that take place in the core and on the surface of the NC, respectively. This leads to NC structures consisting of metallic Au cores with shell structures (also reported in the literature as cages) formed from thiolate-Au bond networks, $-\mathrm{SR}(-\mathrm{Au}-\mathrm{SR})_{n^{-}}$, known as 'staple motifs' $15,16,22$. Divide-and-protect (or similar 'core-in-cage') theory has proven very effective in suggesting NC structural characteristics, with every experimentally identified NC having this core-in-shell structure $^{15,23}$. The superatom theory, on the other hand, states that magic-number stability results from the formation of closedshell electronic orbital structures, similar to noble gases ${ }^{17}$. This theory has been successful in explaining the optical and catalytic properties of several magic-number NCs, but has been shown to have weaknesses as a universal descriptor for the thermodynamic stability of thiolated $\mathrm{Au} \mathrm{NCs}{ }^{14,24}$. In particular, the $\mathrm{Au}_{20} \mathrm{SR}_{16}$ and $\mathrm{Au}_{36} \mathrm{SR}_{24}$ do not fall in the predictions of the superatom theory, but have been successfully experimentally synthesized and isolated under thermodynamic conditions $\mathrm{s}^{25,26}$. In addition, although this theory was originally derived solely based on Au NCs, it should theoretically apply to all metals that fall on the same column of the periodic table, since it applies electron counting and shell closure rules. Yet, metals that fall in the same periodic table column (for example, $\mathrm{Au}$ versus $\mathrm{Cu}$ ) do not form $\mathrm{NCs}$ of the same size (number of metal atoms and ligands) and structure. Moreover, the divide-and-protect theory only suggests structural trends and does not introduce a quantitative descriptor for NC stability, resulting in theoretical predictions of NC structures that are based on general structural criteria. In this context we define structure as composition (Au versus $\mathrm{S}$ content) in addition to NC size and shape (morphology). As a result, the reported computationally predicted NCs have deviated from the experimentally synthesized ones as in the case of $\mathrm{Au}_{24}(\mathrm{SR})_{20}$ (refs 11,12). Beyond firstprinciples calculations, simple geometric scaling laws relating the total number of $\mathrm{Au}$ atoms $(n)$ to the number of ligands $(m)$ in NCs have been discovered, though these relations show limitations in predicting NC morphology ${ }^{27,28}$. In addition, the in silico structural prediction of stable NCs is currently computationally intractable for NC sizes larger than a couple of hundred atoms (treated with first-principles methods). Taking all these observations together, there is a critical need to develop theoretical models able to describe the stability of colloidal NCs as a function of the specific NC structural characteristics.

Herein, we propose a 'thermodynamic stability' theory based on first-principles density functional theory (DFT) calculations performed on experimentally identified metal NCs. Our developed theory introduces thermodynamic descriptors that are dependent on the detailed structural characteristics of the NCs. Moreover, our theory introduces new pathways for discovering in silico atomic-precise metal NC architectures that are thermodynamically stable and synthesizable in the lab.

\section{Results}

Thermodynamic stability theory. Figure 1 highlights all DFT-optimized Au nanostructures along with the designation of which atoms are part of the core or shell. We note that the definition of core and shell metal atoms agrees with previous work ${ }^{25,29-35}$ with the exception of $\mathrm{Au}_{18} \mathrm{SR}_{14}$ and $\mathrm{Au}_{102} \mathrm{SR}_{44}$, where the natural bond orbital charge analysis and S-bonding methods revealed that the core could be more precisely defined by 8 atoms rather than 9 and 77 rather than 79 (see analysis in Supplementary Fig. 2 and Supplementary Note 1$)^{29}$. Based on the core and shell determinations, we calculated the shell-to-core binding energy (BE) and the cohesive energy (CE) of the metal core (see Methods for detailed description). In Fig. 2, we plot the calculated shell-to-core $\mathrm{BE}$ versus the $\mathrm{CE}$ of the cores for the experimentally determined thiolate-protected $\mathrm{Au}$ NCs (points coloured in gold). Interestingly, we reveal a near-perfect match between the shell-to-core $\mathrm{BE}$ and the $\mathrm{CE}$ of the metal cores. This trend highlights a unique physicochemical feature of the experimentally synthesized $\mathrm{Au}_{n}(\mathrm{SR})_{m}$ NCs: in order for a thiolate-protected Au NC to be thermodynamically stable, there is a fine balance between the $\mathrm{CE}$ of the core and the $\mathrm{BE}$ of the shell to the core. Thus, a stability criterion has been elucidated connecting the cores with the shells of the NCs. Interesting enough is the observation that this criterion applies to both neutral (Fig. 1(a)-(i)) and charged (Fig. 2(i)) NCs. In addition, the two structures which would not be identified as stable by the superatom theory, $\mathrm{Au}_{20} \mathrm{SR}_{16}$ and the $\mathrm{Au}_{36} \mathrm{SR}_{24}$, are shown as stable here by the thermodynamic stability theory. To test if our model can be extended to other metals, we performed the same analysis for the $\left[\mathrm{Ag}_{25}\left(\mathrm{SPhMe}_{2}\right)_{18}\right]^{-} \mathrm{NC}$ (Fig. 2(ii)) which has been experimentally synthesized ${ }^{36}$. As shown in Fig. 2, again, the $\mathrm{CE}$ of the core and the $\mathrm{BE}$ of the shell to the core strike a perfect energy balance (see silver point on parity graph). It should be noticed that the $\mathrm{Ag} \mathrm{NC}$ is negatively charged as in the case of the $\left[\mathrm{Au}_{25} \mathrm{SR}_{18}\right]^{-} \mathrm{NC}$ (Fig. 2(i)), verifying not only that this trend holds for different metals, but also when these metals are charged. As an additional validation test, we created the $\left[\mathrm{Cu}_{25} \mathrm{SR}_{18}\right]^{-} \mathrm{NC}$ (Fig. 2(iii)) based on the crystallographic structure of the corresponding $\left[\mathrm{Au}_{25} \mathrm{SR}_{18}\right]^{-} \mathrm{NC}$. It is worth mentioning that according to the superatom theory the $\left[\mathrm{Cu}_{25} \mathrm{SR}_{18}\right]^{-}$should be a thermodynamically stable nanostructure since $\mathrm{Cu}$ and $\mathrm{Au}$ are metals with similar electronic shell closure (same period metals). However, the $\left[\mathrm{Cu}_{25} \mathrm{SR}_{18}\right]^{-}$has not been experimentally synthesized as of yet, and, we note that that according to our model, the $\mathrm{CE}$ of the core dominates the $\mathrm{BE}$ of the shell to the core (red point in Fig. 2 deviating from the parity line). While the challenge with synthesizing $\mathrm{Cu} \mathrm{NCs}$ is largely tied to the persistence of the $\mathrm{Cu}(\mathrm{I})$ state ${ }^{37}$, our calculation imposes the ideal experimental case where the $\mathrm{Cu}$ in $\left[\mathrm{Cu}_{25} \mathrm{SR}_{18}\right]^{-}$remains $\mathrm{Cu}(0)$. Therefore, we suggest that, at least for this ligand configuration (type and number of ligands), the $\left[\mathrm{Cu}_{25} \mathrm{SR}_{18}\right]^{-}$ cannot be a magic number NC. We thus believe that the $\left[\mathrm{Cu}_{25} \mathrm{SR}_{18}\right]^{-}$serves as a case where the core $\mathrm{CE}$ is not balanced with the shell-to-core $\mathrm{BE}$, ruling out this energetic balance as a simple interfacial effect.

To develop a quantitative boundary between synthesizable and non-synthesizable NCs we performed a linear regression on all the experimentally synthesized NCs with $95 \%$ confidence and 

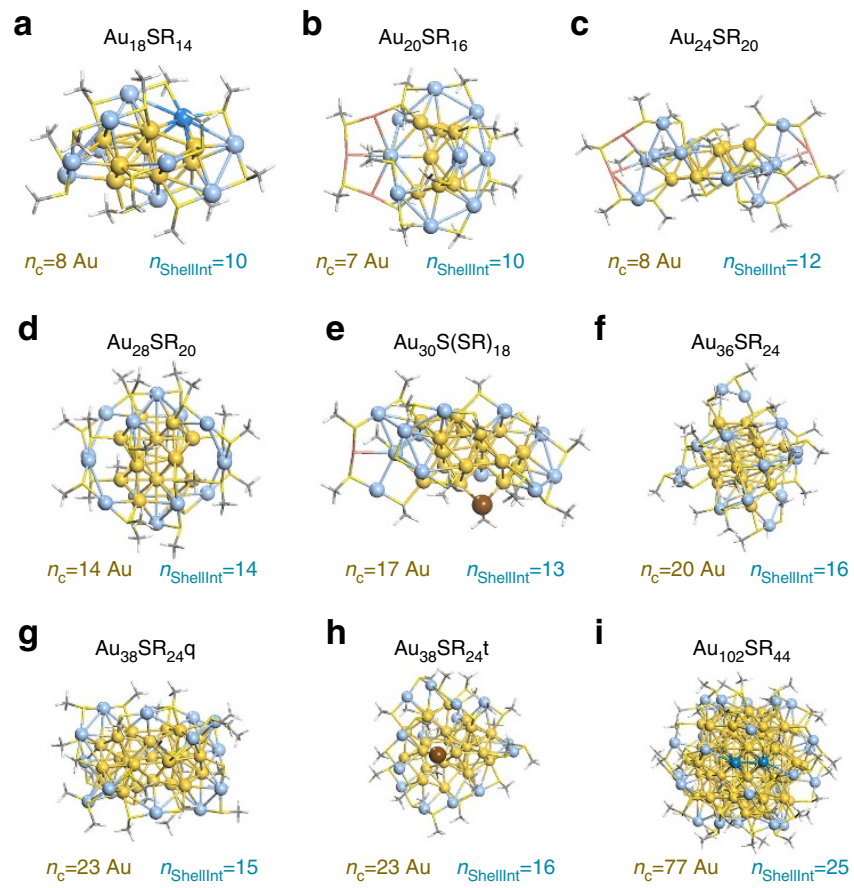

Figure 1 | Optimized geometries of the experimentally synthesized metal nanoclusters. (a) $\mathrm{Au}_{18} \mathrm{SR}_{14}$ (ref. 29), (b) $\mathrm{Au}_{20} \mathrm{SR}_{16}$ (ref. 25), (c) $\mathrm{Au}_{24} \mathrm{SR}_{20}$ (ref. 30), (d) $\mathrm{Au}_{28} \mathrm{SR}_{20}$ (ref. 32), (e) $\mathrm{Au}_{30} \mathrm{~S}(\mathrm{SR})_{18}$ (ref. 19), (f) $\mathrm{Au}_{36} \mathrm{SR}_{24}$ (ref. 33), (g) $\mathrm{Au}_{38} \mathrm{SR}_{24} 9$ (ref. 34), (h) $\mathrm{Au}_{38} \mathrm{SR}_{24}$ (ref. 65) and (i) $\mathrm{Au}_{102} \mathrm{SR}_{44}$ (ref. 35). $n_{c}$ represents the number of core metal atoms while $n_{\text {Shelllnt }}$ represents the number of shell-to-core interactions. Ligands $\left(\mathrm{S}-\mathrm{CH}_{3}\right)$ are shown in stick representation while core and shell atoms, in ball and stick, have been coloured yellow and blue, respectively. In b,c, shell Au atoms which do not interact with the core have been coloured red and are shown in stick representation, while in $\mathbf{a}, \mathbf{i}$ shell Au atoms which were previously identified as core are coloured darker blue. In $\mathbf{e}, \mathbf{h}$, shell sulfur atoms which are not directly bound to a shell Au atom are shown as brown balls.

superimposed the prediction bands (Supplementary Fig. 4). To explore the effectiveness of the $95 \%$ confidence and prediction bands in distinguishing between non-stable and stable NCs we optimized additional hypothetical NCs. Beyond the hypothetical $\left[\mathrm{Cu}_{25} \mathrm{SR}_{18}\right]^{-} \mathrm{NC}$, we investigated the $\mathrm{Ag}_{18} \mathrm{SR}_{14}, \mathrm{Cu}_{18} \mathrm{SR}_{14}$, $\mathrm{Ag}_{38} \mathrm{SR}_{24} q$ and $\mathrm{Cu}_{38} \mathrm{SR}_{24} q$ theoretical NCs generated directly from their corresponding Au NC analogues. We found that they exhibit $\mathrm{CE}$ and $\mathrm{BE}$ values that deviate beyond the $95 \%$ prediction band (Supplementary Fig. 5). In addition, we have tested our method on four theoretically predicted $\mathrm{Au} \mathrm{NCs}$, the $\mathrm{Au}_{18} \mathrm{SR}_{14}$, $\mathrm{Au}_{20} \mathrm{SR}_{16}, \mathrm{Au}_{24} \mathrm{SR}_{20}$ and $\mathrm{Au}_{40} \mathrm{SR}_{24}$, and showed that two $\left(\mathrm{Au}_{24} \mathrm{SR}_{20}\right.$ and $\left.\mathrm{Au}_{40} \mathrm{SR}_{24}\right)$ out of the four exhibit similar deviation from parity as the theoretical Cu NCs, whereas, the $\mathrm{Au}_{18} \mathrm{SR}_{14}$ and $\mathrm{Au}_{20} \mathrm{SR}_{16} \mathrm{NCs}$ exhibit the $\mathrm{CE}$ and $\mathrm{BE}$ energy balance. Therefore, this energetic balance is sensitive to the actual NC structure and the $95 \%$ prediction bands can further be used as cutoffs to screen theoretical NCs predicted with current best practices (Supplementary Fig. 5, Supplementary Note 3$)^{11,38-40}$.

It should be noticed that when switching the thiolate $\mathrm{R}$ group to methyl (to reduce computational cost), attention should be given to the effect that this change introduces to the stability of the shell structure, and in turn, to the shell binding to the core of the NC. Toward understanding ligand impact we highlight that experimentally ${ }^{41}$ and theoretically ${ }^{42}$, the $\left[\mathrm{Au}_{25} \mathrm{SR}_{18}\right]^{-} \mathrm{NC}$ has been shown to be stable for a wide variety of ligands, and was successfully synthesized even with small, ethyl $\mathrm{R}$ groups ${ }^{43}$. Therefore, the exceptional structural stability of $\left[\mathrm{Au}_{25} \mathrm{SR}_{18}\right]^{-}$

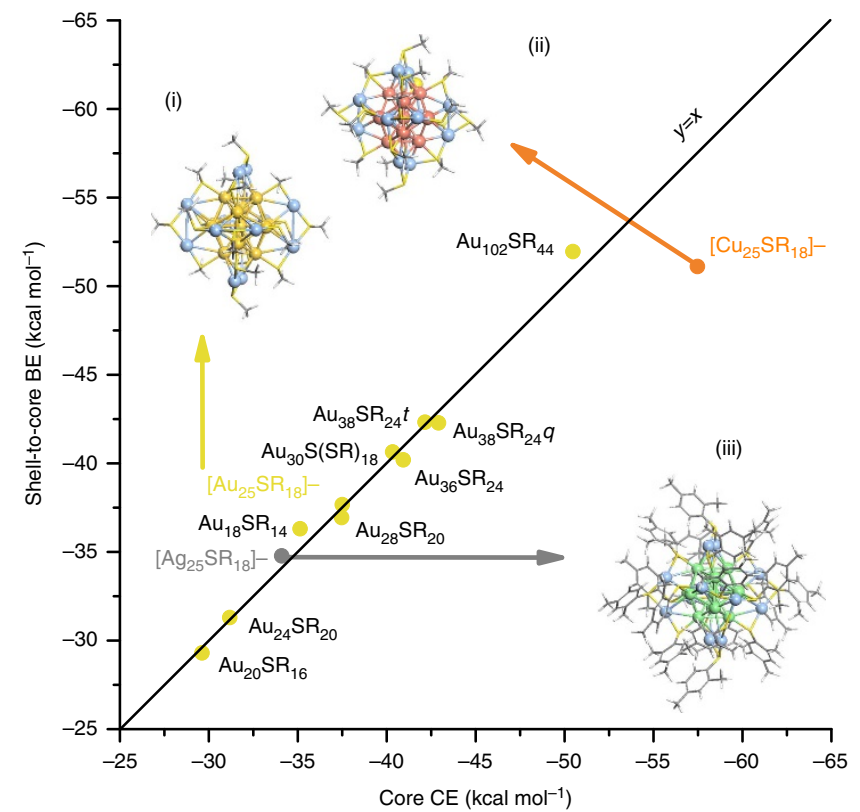

Figure 2 | Parity between core cohesive energy and the shell-to-core BE. The corresponding structures of the $\mathrm{Au}_{n}(\mathrm{SR})_{\mathrm{m}} \mathrm{NCs}$ are presented in Fig. 1 except from the optimized structures of (i) $\left[\mathrm{Au}_{25} \mathrm{SR}_{18}\right]^{-}$(ref. 31),

(ii) $\left[\mathrm{Cu}_{25} \mathrm{SR}_{18}\right]^{-}$and (iii) $\left[\mathrm{Ag}_{25}\left(\mathrm{SPhMe}_{2}\right)_{18}\right]^{-}$(ref. 36) $\mathrm{NCs}$, which are shown as insets in the graph. For $\mathrm{i}-\mathrm{iii}, n_{\mathrm{c}}=13$ metal atoms ( $\left.\mathrm{Au} / \mathrm{Cu} / \mathrm{Ag}\right)$ and $n_{\text {Shelllnt }}=12$ as in Fig. 1. The shell metal atoms are shown in blue, whereas, the $\mathrm{Cu}$ and $\mathrm{Ag}$ core metal atoms are shown in red and green, respectively. Here, all the Au and Ag NCs reported have been experimentally determined. The $\mathrm{Cu} N \mathrm{NC}$ structure is hypothetical, optimized from the Au NC analogous structure (i).

NC seems to be experimentally independent of the ligand type, highlighting the importance of metal structure and $\mathrm{AuS}^{-1}$ stoichiometry in determining stable NCs. For NC structures investigated in this work interactions at the interface between their core and shell regions should be to a large degree unaffected by the ligand selection ${ }^{44}$ (see Supplementary Fig. 6 and Supplementary Note 4 where $\mathrm{Au}_{18} \mathrm{SR}_{14}$ and $\left[\mathrm{Au}_{25} \mathrm{SR}_{18}\right]^{-}$optimization with full ligands resulted to minor energy shifts and for detailed analysis of the $\left[\mathrm{Ag}_{25} \mathrm{SR}_{18}\right]^{-}$case). In addition, metal-metal interactions at the interface are energetically far stronger than the ligand-ligand interactions and capture the core-shell and the relative NC stability. However, enhanced ligand-ligand (R-group) interactions can impact the overall NC stability and associated physicochemical properties as seen in several other recent works $s^{45,46}$. For example, in the case of the $\left[\mathrm{Ag}_{25}\right]^{-} \mathrm{NCs}$, a $\pi$-stacking is observed in the original experimental crystal structures between the phenyl groups present on the shell of the NC. Although the $\mathrm{R}=$ methyl group substitution has been shown to have small effect on the RS-Au bond strength ${ }^{20,47,48}$, a hydrogen-bond network formed at the NC surface by the groups of the ligands, can potentially induce strain on the shell structure, changing in turn the shell-to-core BE (Supplementary Fig. 6). Interestingly, this observation is in agreement with recent work where conversion from $\mathrm{Au}_{38} \mathrm{SR}_{24}(\mathrm{R}=$ phenylethanethiolate) to $\mathrm{Au}_{36} \mathrm{SR}_{24}(\mathrm{R}=4$-tert-butylbenzenethiol) was achieved in solution by swapping the thiolate $\mathrm{R}$ groups from phenylethanethiolate to 4-tert-butylbenzenethiol, altering the hydrogen-bond network formed the surface of the $\mathrm{NCs}^{49}$. To further prove that this structural thermodynamic stabilization is a general behaviour and originates solely from the energy balance between the core and the shell of the NCs we analysed (Supplementary Note 5) CE 
a

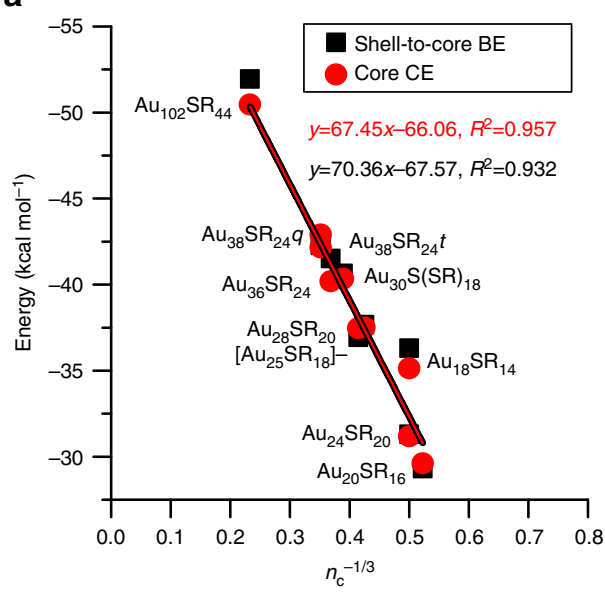

C

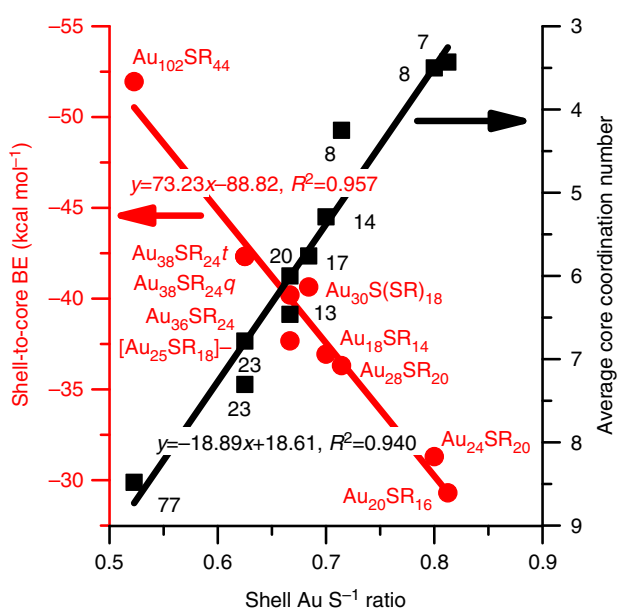

b

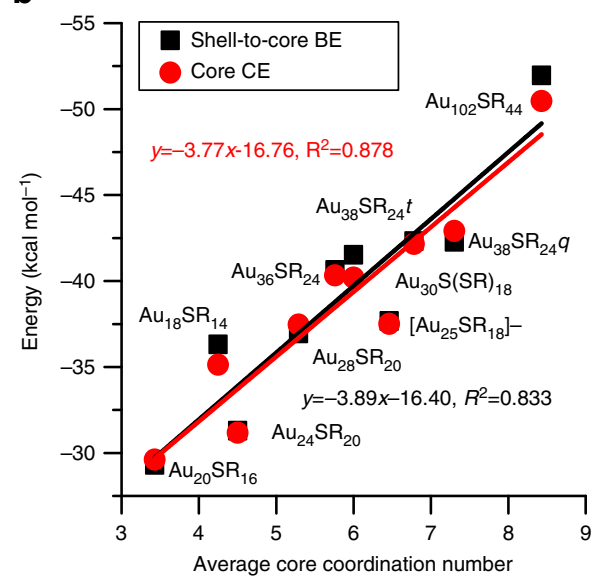

d

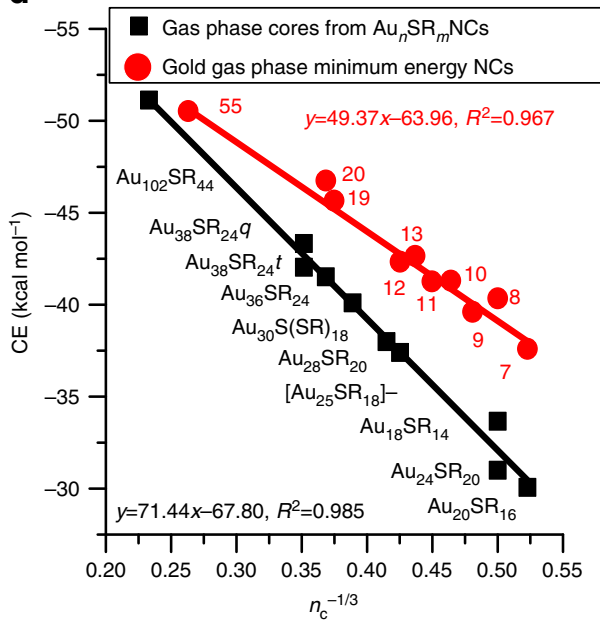

Figure 3 | Nanocluster stability-morphology relations. (a) Core CE and shell-to-core BE versus $n_{c}^{-1 / 3}$ (number of core metal atoms) for cores of thermodynamically stable magic number Au NCs. (b) Core CE and shell-to-core BE versus average coordination numbers (CNs) for cores of Au NCs. (c) Shell-to-core $\mathrm{BE}$ and average core $\mathrm{CN}$ versus the ratio of total $\mathrm{Au}$ atoms and $\mathrm{S}$ atoms in the shells and (d) global minima gas phase Au clusters and cores of $\mathrm{Au} \mathrm{NCs}$. From Figs 1 and 2, the Au NC cores contain: $\mathrm{Au}_{18} \mathrm{SR}_{14}=8, \mathrm{Au}_{20} \mathrm{SR}_{16}=7, \mathrm{Au}_{24} \mathrm{SR}_{20}=8,\left[\mathrm{Au}_{25} \mathrm{SR}_{18}\right]^{-}=13, \mathrm{Au}_{28} \mathrm{SR}_{14}=14$, $\mathrm{Au}_{30} \mathrm{~S}(\mathrm{SR})_{14}=17, \mathrm{Au}_{36} \mathrm{SR}_{24}=20, \mathrm{Au}_{38} \mathrm{SR}_{24} q, t=23$ and $\mathrm{Au}_{102} \mathrm{SR}_{44}=77$ Au atoms.

and $\mathrm{BE}$ in the presence of the common ${ }^{8}$ dichloromethane (Supplementary Fig. 7(i)) and water (Supplementary Fig. 7(ii)) solvents. The parity between core $\mathrm{CE}$ and shell-to-core $\mathrm{BE}$ was maintained, with the solvent only weakly affecting the shell-tocore BE. Moreover, we have also tested different DFT methods on a randomly selected system and found that the parity between the core $\mathrm{CE}$ and shell-to-core $\mathrm{BE}$ were maintained with very slight deviations (Supplementary Table 1). Finally, we present a detailed thermodynamic analysis in Supplementary Note 6 on how this energy balance between the core and the shell relates to the total chemical potential change of the $\mathrm{NC}(\Delta \mu(\mathrm{NC})=0$ at equilibrium), rationalizing the importance of these descriptors and the thermodynamic stability theory.

Nanocluster size and shape relations. Because the developed thermodynamic stability theory is based on the morphologydependent energetic factors of $\mathrm{CE}$ of the core and the $\mathrm{BE}$ of the shell to the core, we expect these properties to correlate with the structural characteristics of the NCs (that is, size and shape). For example, it is well known that the CE of metals scale linearly with $n_{\mathrm{m}}^{-1 / 3}$, where $n_{\mathrm{m}}$ is the number of metal atoms in a pure metal cluster. Actually, one can apply first-principles calculations to derive such linear trends, the limit of which shows the $\mathrm{CE}$ of the bulk, when $n_{\mathrm{m}} \rightarrow \infty$, as has been shown in the case of $\mathrm{Au}$ (refs 50,51). In Fig. 3a we present such an analysis (core CE versus $n_{\mathrm{c}}^{-1 / 3}$, where $n_{\mathrm{c}}$ is the number of Au atoms in the core of the NCs) and superimpose the shell-to-core $\mathrm{BE}$ results, highlighting the linearity between both energetic factors with $n_{\mathrm{c}}^{-1 / 3}$ for the thermodynamically stable Au NCs. The reason $n_{\mathrm{c}}^{-1 / 3}$ trends linearly with $\mathrm{CE}$ is attributed to the decrease in the fraction of low-coordinated (surface) sites observed on the NCs as the cluster size increases ${ }^{51}$. Surprisingly, the shell-to-core BE was also found to scale linearly with $n_{\mathrm{c}}^{-1 / 3}$, with almost identical behaviour (see linear fits) as the CE. The identification of a common structural descriptor for the $\mathrm{CE}$ and the shell-to-core $\mathrm{BE}$ behaviour on the NCs helps rationalize the observed parity between these two energy contributions in Fig. 2. Since the $n_{c}^{-1 / 3}$ shows how the low-coordinated sites scale with NC size (number of metal atoms, $n_{\mathrm{c}}$ ), then we should expect that the average coordination number $(\mathrm{CN})$ to scale linearly as well with both the $\mathrm{CE}$ and the shell-to-core BE. This behaviour is clearly demonstrated in Fig. 3b. The average $\mathrm{CN}$ on Au can practically range from 0 (atom) to 12 (bulk). As the average $\mathrm{CN}$ of the $\mathrm{NC}$ increases, the $\mathrm{CE}$ increases (more exothermic values) because the $\mathrm{Au}$ atoms tend to form more bonds with their neighbours, 
a

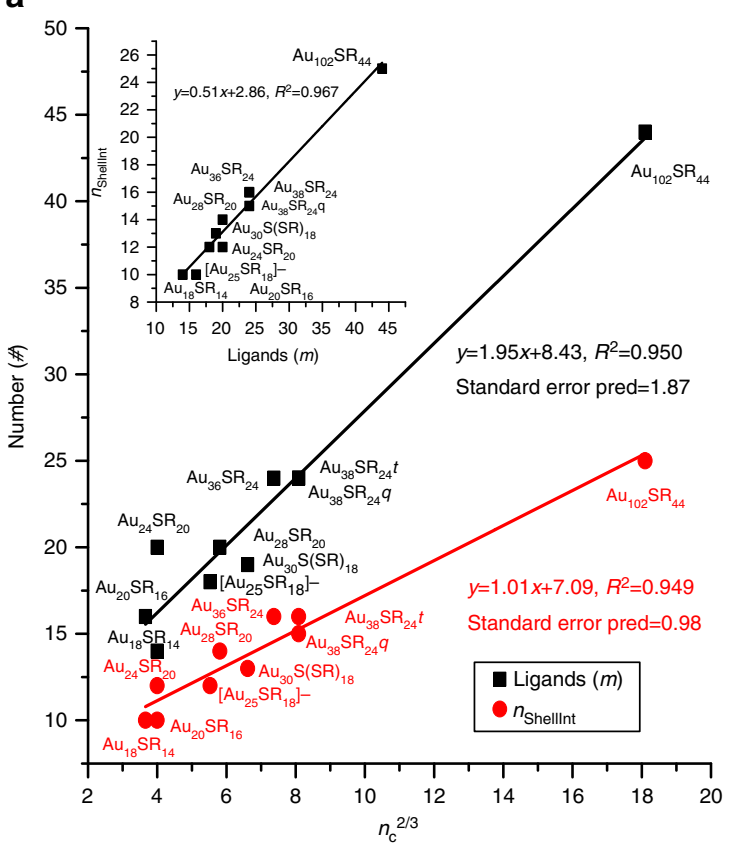

b

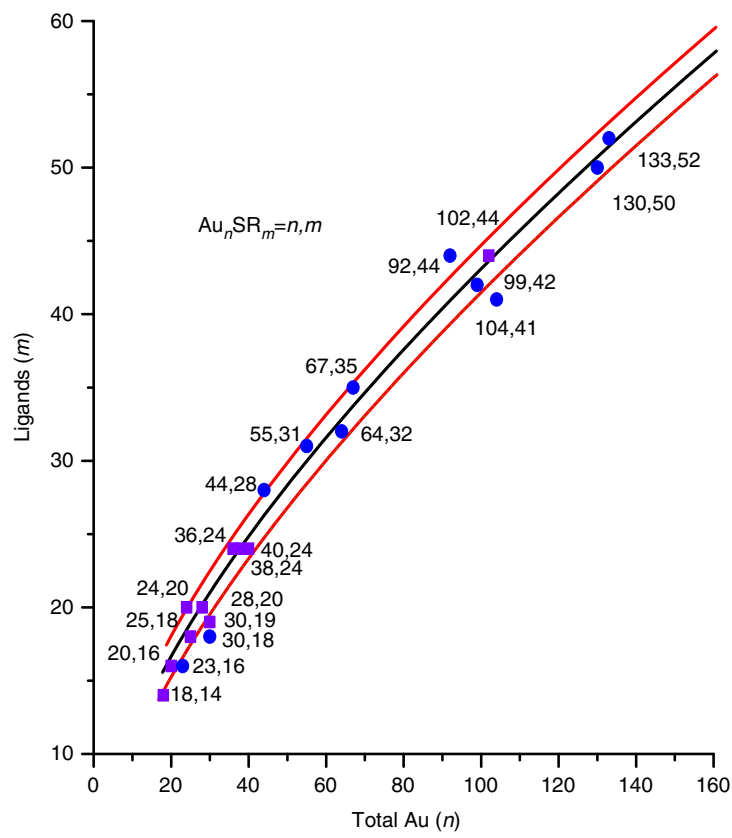

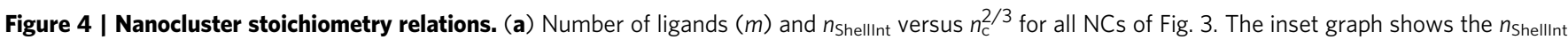
versus $m$ behaviour. (b) Predicted stoichiometric trend between number of ligands $(m)$ and total $A u(n)$ atoms of the NCs. The predictions were made using the relations shown in $\mathbf{a}$. The black line represents the best fit, whereas, the surrounding red lines the standard error in the prediction. The purple square points represent experimentally stable NCs used in our calculations to develop the model, whereas, the blue circles represent other experimentally stable NCs identified in literature ${ }^{8}$.

increasing the overall stability of the NC. On the other hand, as we have recently shown in the area of catalysis, the adsorbates show higher BEs (more exothermic) on sites of the NCs with low $\mathrm{CNs}^{52,53}$. However, this is not the case here (see red point data in Fig. 3b). The thiolated-Au shell network binds the core in a way that is counterintuitive to the common belief: as the average $\mathrm{CN}$ of the NC increases, the adsorption strength of the shell increases as well. This counterintuitive trend is highlighted by the difference between the predicted and experimental core structures (and resulting deviation from the parity plot) in the $\mathrm{Au}_{24} \mathrm{SR}_{20} \mathrm{NC}$, where the experimental structure showed a core with lower $\mathrm{CN}$ than the predicted structure (Supplementary Fig. 5) ${ }^{11,30}$. In Fig. $3 \mathrm{c}$ we plot the shell-to-core BE versus the shell $n_{\mathrm{Au}} n_{\mathrm{S}}^{-1}$ ratio (red circles), where $n_{\mathrm{Au}}$ is the number of shell $\mathrm{Au}$ atoms and $n_{\mathrm{S}}$ is the number of sulfur atoms on the shell (equivalent to $m$ in $\mathrm{Au}_{\mathrm{n}} \mathrm{SR}_{\mathrm{m}}$ ). The shell $n_{\mathrm{Au}} n_{\mathrm{S}}^{-1}$ ratio demonstrates the cationic character degree of $\mathrm{Au}$ on the shell of the $\mathrm{NC}\left(\mathrm{SR}^{\delta-}\right.$ interacting with $\left.\mathrm{Au}^{\delta+}\right)$ and concentration of bridging thiol groups (SR groups not directly bound to the cores). On the same graph, we plot the average $\mathrm{CN}$ of the $\mathrm{NC}$ cores versus the $n_{\mathrm{Au}} n_{\mathrm{S}}^{-1}$ ratio (black rectangles) on the shell. Notice that both the shell-to-core $\mathrm{BE}$ and the core $\mathrm{CN}$ scale linearly with the shell $n_{\mathrm{Au}} n_{\mathrm{S}}^{-1}$ ratio. It can be observed that the lower the $n_{\mathrm{Au}} n_{\mathrm{S}}^{-1}$ ratio, the stronger the shell-to-core $\mathrm{BE}$ because of both the increased electrostatic interactions between the core and shell $\mathrm{Au}$ atoms (latter are charged more positively) and the decreased amount of bridging thiol groups, which tend to pull shell $\mathrm{Au}$ atoms away from the core ${ }^{47}$. On the other hand, the $\mathrm{CN}$ versus shell $n_{\mathrm{Au}} n_{\mathrm{S}}^{-1}$ ratio linear trend has a negative slope compared to the shell-to-core $\mathrm{BE}$ versus $n_{\mathrm{Au}} n_{\mathrm{S}}^{-1}$ ratio linear trend. This fact explains why the shell-to-core BE was found to counterintuitively increase as the average core $\mathrm{CN}$ increases. This observation was made based on the $\mathrm{Au}$ to SR stoichiometry in only the shells of the NCs. Examining the total $\mathrm{Au}$ to SR ratio on the entire $\mathrm{NC}$, we note an overall agreement with the experimental observation of increasing $\mathrm{NC}$ diameter resulting from increasing $\mathrm{Au}$ to $\mathrm{SR}$ ratio in solution $^{54}$.

In Fig. 3d, we show the gas phase $\mathrm{CE}$ versus $n_{\mathrm{c}}^{-1 / 3}$ trend for the $\mathrm{Au}_{n} \mathrm{SR}_{m}$ core structures (without the presence of the shells) identified from the crystal structures of the experimentally synthesized NCs (black rectangles) and compare against the $\mathrm{CE}$ behaviour of the global minimum energy gas phase Au NC structures of the same size range (red circles). Interestingly, the gas phase CE (equation (3)) is roughly equivalent to the core CE calculated with the presence of the shells in the NCs (see equation (2) and Supplementary Fig. 8). Therefore, the gas phase CEs of the NC cores, accurately represent the stability of the cores in the NC (presence of shell), and can be directly compared with the gas phase global minimum energy structures, in Fig. 3d. The initial structures of the global minimum gas phase clusters were taken from recent literature and were optimized at the same level of theory as the NC cores ${ }^{55-57}$. Figure $3 \mathrm{~d}$ reveals a difference in the slopes between the minimum energy NCs and the core NC structures. The difference in slopes can be attributed to the morphology imposed on the Au NC cores by the presence of the thiolate shell. Notice that gas phase minimum energy $\mathrm{Au}$ clusters preferentially obtain planar structures up to $\mathrm{Au}_{13}$, whereas, in the presence of the metal-thiolate shell, they obtain threedimensional structures ${ }^{55,58}$. We believe that other magic-number thiolated $\mathrm{Au} \mathrm{NC}$ cores will fall directly on the black line. Overall, Fig. 3 demonstrates for the first time that the stabilization of colloidal NCs in solution is dictated by two thermodynamic descriptors that need to balance: the metal core of the NC tends to grow to increase the $\mathrm{CE}$ with $\mathrm{NC}$ size (descriptor: $\mathrm{CE}$ ), while the thiolate-Au network on the shell (acting as adsorbates) obtains a specific composition in staple motifs $\left(n n_{\mathrm{S}}^{-1}\right.$ ratio), 
tuning the shell-to-core $\mathrm{BE}$ to match the $\mathrm{CE}$ of the core at each NC size.

Nanocluster stoichiometry relations. Moving forward, using these relations discovered in Fig. 3, additional stoichiometry rules are needed (that is, $\mathrm{Au}_{n} \mathrm{SR}_{m}$ stoichiometries in addition to core and shell information) to construct a useful methodology for NC prediction. Toward stoichiometry prediction, previous work identified a geometric descriptor based on the surface area to volume ratio of the NCs that relates the number of ligands $(m)$ to the total number of $\mathrm{Au}$ atoms $(n)$ in the NCs with a linear trend of $m$ versus $n^{2 / 3}$ (refs 27,28). For the NCs $n \approx n_{\mathrm{c}}+n_{\text {ShellInt }}$ (very small deviations can occur when a shell $\mathrm{Au}$ does not bind the core, or a $\mathrm{S}$ atom is a direct contact to the core). Given that $n_{\text {ShellInt }} \approx n-n_{\mathrm{c}}$ and the $\mathrm{AuS}^{-1}$ ratio in the shell dictates a linear trend with shell-to-core BE (Fig. 3c) we would expect $m$ and $n_{\text {ShellInt }}$ to be correlated. The inset of Fig. 4 a shows that $m$ scales perfectly linearly with $n_{\text {ShellInt }}\left(R^{2}=0.967\right)$. Since $m$ and $n_{\text {ShellInt }}$, and $m$ and $n^{2 / 3}$, are linearly related and since $n_{\mathrm{c}} \approx n-n_{\text {ShellInt }}$, a $2 / 3$ exponential relationship (predominates linear functionality) also exists between $n_{\mathrm{c}}$ and $m$ (Fig. 4a). As a result, these observations establish a parametric model for $n$ and $m$ founded on $n_{\text {c }}$. This parametric model, which can now predict the overall NC stoichiometry, is presented in Fig. 4b. We have thus shown (using the relations derived from Fig. 3) that the core morphology largely dictates the overall NC characteristics. Along these lines, our new model captures the previously identified $m$ versus $n^{2 / 3}$ behaviour and nearly all of the NCs fall within the $95 \%$ prediction intervals. Because this model is parametric with $n_{\mathrm{c}}$, however, specific core and shell region information can be immediately derived for NCs of any given $n, m$. For example, given $n_{\mathrm{c}}=45$, $m \approx 32-34$ and $n_{\text {ShellInt }} \approx 19-20$ resulting in the $\mathrm{Au}_{64} \mathrm{SR}_{32}$, $\mathrm{Au}_{65} \mathrm{SR}_{34}$, and any other combination between these $n, m$ values to identify NCs (see Fig. 4b for experimentally synthesized $\mathrm{Au}_{64} \mathrm{SR}_{32} \mathrm{NC}$ ). From this point, the structure-energy relationships identified in Fig. 3 can be used to feed further structural information to the NC prediction, such as the core $\mathrm{CN}$, as well as to screen candidate structures based on the energy balance criterion between the core CE and the shell-to-core BE (Fig. 2). Thus, the identified relationships aid the prediction of NCs that span sizes larger than the ones currently affordable by high-throughput DFT calculations ${ }^{18}$.

\section{Discussion}

In summary, we present a thermodynamic stability theory derived from first-principles calculations, rationalizing the stability of colloidal metal NCs in solution and significantly advancing the previously proposed divide-and-protect and superatom theories ${ }^{15,17}$. Our theory reveals that for every thermodynamically isolated, experimentally synthesized thiolate-protected $\mathrm{NC}$, there is a perfect energy balance between the adsorption strength of the ligand-shell to the metal-core and the CE of the core. Our theory applies to both neutral and charged NCs, as well as to different metals. In addition, we highlight the impact of the thiolate ligands on the overall stability and size/shape of the $\mathrm{NC}^{5}$. Finally, this theory directly relates these thermodynamic stability (energy) contributions to geometrical characteristics of metal cores of the NC, rationalizing NC size and shape effects on NC stability and opening new avenues for in silico NC predictions.

\section{Methods}

$\boldsymbol{A} \boldsymbol{b}$ initio methodology. We used the BP-86 (refs 59,60) functional combined with the def2-SV(P) basis set ${ }^{61}$ accelerated with the resolution of identities approximation $^{62,63}$ as implemented in the Turbomole 6.6 package ${ }^{64}$. Structures were taken directly from previously published work and the $\mathrm{R}$ groups of the thiolates were substituted by methyl groups $6,11,19,25,29-33,35,38-40,65$. conductor-like screening model (COSMO) implicit solvation models were also employed to gauge the effect of dichloromethane $(\varepsilon=8.93)$ solvent on the developed model ${ }^{66,67}$. The BP-86 functional has been successfully used on thiolated-metal NC systems ${ }^{48,68}$ and the $\mathrm{R}=$ methyl group substitution has had little impact on RS-Au bond strength as has been previously applied in computational NC structural determinations ${ }^{20,47,48}$. We also note that BP-86 has been successful in capturing stability trends and cohesive energies of very small pure gold clusters ${ }^{69}$. We did not include van der waals corrections in our calculations as they tend to overestimate $\mathrm{Au}-\mathrm{Au}$ bonding at the interface of $\mathrm{Au}-$ thiolate layers ${ }^{44}$. All optimizations were performed without any symmetry constraints.

Definition of shell-to-core BE. Two methods were used to identify if Au atoms were 'core' or 'shell', that of natural bond orbital charge analysis and that of measuring S-contacts of $\mathrm{Au}$ atoms in the structure, where the shell $\mathrm{Au}$ atoms have exactly two bonded sulfur groups (see Supplementary Fig. 1 for example of $\mathrm{Au}_{20}$ determination $)^{70}$. These two methods were in perfect agreement over all NCs. With core and shell designations, we isolated the core and shell sections of the NCs and performed single point energy calculations on each section. From the (1) optimized NC structure, (2) separated core and (3) separated shell results, the shell-to-core BE and core $\mathrm{CE}$ were calculated. The shell-to-core $\mathrm{BE}$ is defined as:

$$
\text { Shell }- \text { to }- \text { Core BE }=\frac{E_{\text {Full Cluster }}-E_{\text {Shell }}-E_{\text {Core }}}{n_{\text {ShellInt }}}
$$

where $E_{\mathrm{x}}=$ electronic energy of group $\mathrm{X}$ and $n_{\text {Shellint }}=$ number of shell contacts interacting with the core (Figs 1 and 2 ). $n_{\text {ShellInt }}$ is largely dictated by the number of shell $\mathrm{Au}$ atoms in contact with the surface of the cores $(<4 \AA$ from the nearest core $\mathrm{Au}$ atom) because metal-metal bonds dominate the shell-to-core $\mathrm{BE}$

(Supplementary Fig. 3, Supplementary Note 2). Beyond shell Au contacts to the cores, SR groups that are not bound to any shell Au but are bound directly to core Au represent a direct shell-to-core contact and thus are also included in $n_{\text {Shellint }}$ (see Supplementary Note 1 for details surrounding the calculation of $n_{\text {ShellInt }}$ ).

Definition of core CE. The core CE is defined as:

$$
\text { Core CE }=\frac{E_{\text {Full Cluster }}-n_{\mathrm{c}} E_{\text {Metal Atom }}-E_{\text {Shell }}}{n_{\mathrm{c}}+n_{\text {ShellInt }}}
$$

where $n_{\mathrm{c}}=$ number of metal atoms contained in the core structures (and $E$ is the total electronic energy). For each of the core structures different multiplicities were tested and the lowest-energy spin states were selected for the core CE calculation. For the gas phase minimum energy clusters and NC core structures the CE is defined:

$$
\mathrm{CE}=\frac{E_{\text {Cluster }}-n_{\mathrm{c}} E_{\text {Metal Atom }}}{n_{\mathrm{c}}}
$$

For the core structures, Lennard-Jones radii were used to determine the CNs.

Data availability. The datasets generated during and/or analysed during the current study are available from the corresponding author on reasonable request.

\section{References}

1. Daniel, M. C. \& Astruc, D. Gold nanoparticles: assembly, supramolecular chemistry, quantum-size-related properties, and applications toward biology, catalysis, and nanotechnology. Chem. Rev. 104, 293-346 (2004).

2. Sardar, R., Funston, A. M., Mulvaney, P. \& Murray, R. W. Gold nanoparticles: past, present, and future ${ }^{\dagger}$. Langmuir 25, 13840-13851 (2009).

3. Zhu, Y., Qian, H., Drake, B. A. \& Jin, R. Atomically precise $\mathrm{Au}_{25}(\mathrm{SR})_{18}$ nanoparticles as catalysts for the selective hydrogenation of alpha,betaunsaturated ketones and aldehydes. Angew. Chem. Int. Ed. Engl. 49, 1295-1298 (2010).

4. Brust, M., Walker, M., Bethell, D., Schiffrin, D. J. \& Whyman, R. Synthesis of thiol-derivatised gold nanoparticles in a two-phase liquid-liquid system. J. Chem. Soc. Chem. Commun. 801-802 (1994).

5. Chen, Y., Zeng, C., Kauffman, D. R. \& Jin, R. Tuning the magic size of atomically precise gold nanoclusters via isomeric methylbenzenethiols. Nano Lett. 15, 3603-3609 (2015).

6. Jadzinsky, P. D., Calero, G., Ackerson, C. J., Bushnell, D. A. \& Kornberg, R. D. Structure of a thiol monolayer-protected gold nanoparticle at $1.1 \AA$ resolution. Science 318, 430-433 (2007).

7. Aikens, C. M. Electronic structure of ligand-passivated gold and silver nanoclusters. J. Phys. Chem. Lett. 2, 99-104 (2011).

8. Jin, R. Atomically precise metal nanoclusters: stable sizes and optical properties Nanoscale 15, 1549-1565 (2015).

9. Jin, R. et al. Size focusing: a methodology for synthesizing atomically precise gold nanoclusters. J. Phys. Chem. Lett. 1, 2903-2910 (2010).

10. Liu, C., Pei, Y., Sun, H. \& Ma, J. The nucleation and growth mechanism of thiolate-protected Au nanoclusters. J. Am. Chem. Soc. 137, 15809-15816 (2015). 
11. Pei, Y. et al. Interlocked catenane-like structure predicted in $\mathrm{Au}_{24}(\mathrm{SR})_{20}$ : implication to structural evolution of thiolated gold clusters from homoleptic gold(I) thiolates to core-stacked nanoparticles. J. Am. Chem. Soc. 134, 3015-3024 (2012)

12. Pei, Y. \& Zeng, X. C. Investigating the structural evolution of thiolate protected gold clusters from first-principles. Nanoscale 4, 4054-4072 (2012).

13. Mpourmpakis, G., Caratzoulas, S. \& Vlachos, D. G. What controls Au nanoparticle dispersity during growth? Nano Lett. 10, 3408-3413 (2010).

14. Häkkinen, H. Atomic and electronic structure of gold clusters: understanding flakes, cages and superatoms from simple concepts. Chem. Soc. Rev. 37, 1847 (2008).

15. Häkkinen, H., Walter, M. \& Grönbeck, H. Divide and protect: capping gold nanoclusters with molecular gold-thiolate rings. J. Phys. Chem. B 110, 9927-9931 (2006).

16. Iwasa, T. \& Nobusada, K. Theoretical investigation of optimized structures of thiolated gold cluster $\left[\mathrm{Au}_{25}\left(\mathrm{SCH}_{3}\right)_{18}\right]^{+}$. J. Phys. Chem. 111, 45-49 (2007)

17. Walter, M. et al. A unified view of ligand-protected gold clusters as superatom complexes. Proc. Natl Acad. Sci. USA 105, 9157-9162 (2008).

18. Häkkinen, H. The gold-sulfur interface at the nanoscale. Nat. Chem. 4, 443-455 (2012).

19. Crasto, D., Malola, S., Brosofsky, G., Dass, A. \& Häkkinen, H. Single crystal XRD structure and theoretical analysis of the chiral $\mathrm{Au}_{30} \mathrm{~S}(\mathrm{~S}-\mathrm{t}-\mathrm{Bu})_{18}$ cluster. J. Am. Chem. Soc. 136, 5000-5005 (2014).

20. Pei, Y., Gao, Y. \& Zeng, X. C. Structural prediction of thiolate-protected $A_{3}$ : a face-fused bi-icosahedral. J. Am. Chem. Soc. 130, 7830-7832 (2008).

21. Gao, Y., Shao, N. \& Zeng, X. C. Ab initio study of thiolate-protected $\mathrm{Au}_{102}$ nanocluster. ACS Nano 2, 1497-1503 (2008).

22. Yamazoe, S. et al. Hierarchy of bond stiffnesses within icosahedral-based gold clusters protected by thiolates. Nat. Commun. 7, 10414 (2016).

23. Jung, J., Kang, S. \& Han, Y.-K. Ligand effects on the stability of thiol-stabilized gold nanoclusters: $\mathrm{Au}_{25}(\mathrm{SR})_{18}^{-}, \mathrm{Au}_{38}(\mathrm{SR})_{24}$, and $\mathrm{Au}_{102}(\mathrm{SR})_{44}$. Nanoscale 4, 4206-4210 (2012).

24. Reimers, J. R., Wang, Y., Cankurtaran, B. O. \& Ford, M. J. Chemical analysis of the superatom model for sulfur-stabilized gold nanoparticles. J. Am. Chem. Soc, 132, 8378-8384 (2010).

25. Zeng, C., Liu, C., Chen, Y., Rosi, N. L. \& Jin, R. Gold-thiolate ring as a protecting motif in the $\mathrm{Au}_{20}(\mathrm{SR})_{16}$ nanocluster and implications. J. Am. Chem. Soc. 20, 16-19 (2014)

26. Das, A. et al. Cyclopentanethiolato-protected $\mathrm{Au}_{36}\left(\mathrm{SC}_{5} \mathrm{H}_{9}\right)_{24}$ nanocluster: crystal structure and implications for the steric and electronic effects of ligand. J. Phys. Chem. A 36, 8264-8269 (2014).

27. Kimura, K. et al. Size determination of gold clusters by polyacrylamide gel electrophoresis in a large cluster region. J. Phys. Chem. C 113, 14076-14082 (2009).

28. Dass, A. Nano-scaling law: geometric foundation of thiolated gold nanomolecules. Nanoscale 4, 2260-2263 (2012).

29. Das, A. et al. Structure determination of $\left[\mathrm{Au}_{18}(\mathrm{SR})_{14}\right]$. Angew. Chemie Int. Ed. Engl. 54, 3140-3144 (2015)

30. Das, A. et al. Crystal structure and electronic properties of a thiolate-protected $\mathrm{Au}_{24}$ nanocluster. Nanoscale 6, 6458-6462 (2014).

31. Zhu, M., Aikens, C. M., Hollander, F. J., Schatz, G. C. \& Jin, R. Correlating the crystal structure of a thiol-protected $\mathrm{Au}_{25}$ cluster and optical properties. J. Am. Chem. Soc. 130, 5883-5885 (2008).

32. Zeng, C., Li, T., Das, A., Rosi, N. L. \& Jin, R. Chiral structure of thiolateprotected 28 -gold-atom nanocluster determined by X-ray crystallography. J. Am. Chem. Soc. 135, 10011-10013 (2013).

33. Zeng, C. et al. Total structure and electronic properties of the gold nanocrystal $\mathrm{Au}_{36}(\mathrm{SR})_{24}$. Angew. Chem. Int. Ed. Engl. 51, 13114-13118 (2012).

34. Qian, H., Eckenhoff, W. T., Zhu, Y., Pintauer, T. \& Jin, R. Total structure determination of thiolate-protected $\mathrm{Au}_{38}$ nanoparticles. J. Am. Chem. Soc. 132, 8280-8281 (2010).

35. Li, Y., Galli, G. \& Gygi, F. Electronic structure of thiolate-covered gold nanoparticles: $\mathrm{Au}_{102}(\mathrm{MBA})_{44}$. ACS Nano 2, 1896-1902 (2008).

36. Joshi, C. P., Bootharaju, M. S., Alhilaly, M. J. \& Bakr, O. M. $\left[\mathrm{Ag}_{25}(\mathrm{SR})_{18}\right]^{-}$: the 'Golden' silver nanoparticle. J. Am. Chem. Soc. 137, 11578-11581 (2015).

37. Nguyen, T. A. D. et al. A $\mathrm{Cu}_{25}$ nanocluster with partial $\mathrm{Cu}(0)$ character. J. Am. Chem. Soc. 137, 13319-13324 (2015).

38. Tang, Q. \& Jiang, D. E. Revisiting structural models for $\mathrm{Au}_{18}(\mathrm{SR})_{14}$. J. Phys. Chem. C 119, 2904-2909 (2015).

39. Malola, S. et al. $\mathrm{Au}_{40}(\mathrm{SR})_{24}$ cluster as a chiral dimer of 8-electron superatoms: structure and optical properties. J. Am. Chem. Soc. 40, 26-29 (2012).

40. Pei, Y., Gao, Y., Shao, N. \& Xiao, C. Z. Thiolate-protected $\mathrm{Au}_{20}(\mathrm{SR})_{16}$ cluster: prolate $\mathrm{Au}_{8}$ core with new $\left[\mathrm{Au}_{3}(\mathrm{SR})_{4}\right]$ staple motif. J. Am. Chem. Soc. 131, 13619-13621 (2009).

41. Yuan, X., Goswami, N., Mathews, I., Yu, Y. \& Xie, J. Enhancing stability through ligand-shell engineering: a case study with $\mathrm{Au}_{25}(\mathrm{SR})_{18}$ nanoclusters. Nano Res. 8, 3488-3495 (2015).
42. Crasto, D. et al. $\mathrm{Au}_{24}(\mathrm{SAdm})_{16}$ nanomolecules: X-ray crystal structure, theoretical analysis, adaptability of adamantane ligands to form $\mathrm{Au}_{23}(\mathrm{SAdm})_{16}$ and $\mathrm{Au}_{25}(\mathrm{SAdm})_{16}$, and its relation to $\mathrm{Au}_{25}(\mathrm{SR})_{18}$. J. Am. Chem. Soc. 136, 14933-14940 (2014).

43. Pan, F. et al. $\mathrm{Au}_{25}(\mathrm{SEt})_{18}$, a nearly naked structural analysis by single crystal $\mathrm{X}$-ray crystallography and electron nuclear double resonance. ACS Nano $\mathbf{2 5}$, 3904-3912 (2014).

44. Reimers, J. R., Ford, M. J., Halder, A., Ulstrup, J. \& Hush, N. S. Gold surfaces and nanoparticles are protected by $\mathrm{Au}(0)$-thiyl species and are destroyed when $\mathrm{Au}(\mathrm{I})$-thiolates form. Proc. Natl Acad. Sci. USA 113, E1424-E1433 (2016).

45. Chen, Y. et al. Isomerism in $\mathrm{Au}_{28}(\mathrm{SR})_{20}$ nanocluster and stable structures. J. Am. Chem. Soc. 138, 1482-1485 (2016).

46. Heinecke, C. L. et al. Structural and theoretical basis for ligand exchange on thiolate monolayer protected gold nanoclusters. J. Am. Chem. Soc. 134, 13316-13322 (2012).

47. Grönbeck, H., Häkkinen, H. \& Whetten, R. L. Gold-thiolate complexes form a unique $\mathrm{c}(4 \times 2)$ structure on $\mathrm{Au}(111)$. J. Phys. Chem. C 111, 15940-15942 (2008).

48. Barngrover, B. M. \& Aikens, C. M. Oxidation of gold clusters by thiols. J. Phys. Chem. A 117, 5377-5384 (2013).

49. Zeng, C., Chen, Y., Das, A. \& Jin, R. Transformation chemistry of gold nanoclusters: from one stable size to another. J. Phys. Chem. Lett. 6, 2976-2986 (2015).

50. Chan, B. \& Yim, W.-L. Accurate computation of cohesive energies for small to medium-sized gold clusters. J. Chem. Theory Comput. 9, 1964-1970 (2013).

51. Haberlen, O. D., Chung, S. C., Stener, M. \& Rosch, N. From clusters to bulk: a relativistic density functional investigation on a series of gold clusters $\mathrm{Au}_{\mathrm{n}}$, $\mathrm{n}=6$, ..., 147. J. Chem. Phys. 106, 5189-5201 (1997).

52. Mpourmpakis, G., Andriotis, A. N. \& Vlachos, D. G. Identification of descriptors for the $\mathrm{CO}$ interaction with metal nanoparticles. Nano Lett. 10, 1041-1045 (2010).

53. Taylor, M. G., Austin, N., Gounaris, C. \& Mpourmpakis, G. Catalyst design based on morphology and environment dependent adsorption on metal nanoparticles. ACS Catal. 5, 6296-6301 (2015).

54. Hostetler, M. J. et al. Alkanethiolate gold cluster molecules with core diameters from 1.5 to $5.2 \mathrm{~nm}$ : core and monolayer properties as a function of core size. Langmuir 14, 17-30 (1998).

55. Deka, A. \& Deka, R. C. Structural and electronic properties of stable $\mathrm{Au}_{n}$ $(n=2-13)$ clusters: a density functional study. J. Mol. Struct. TheoChem 870, 83-93 (2008)

56. Gruene, P. et al. Structures of neutral $\mathrm{Au}_{7}, \mathrm{Au}_{19}$, and $\mathrm{Au}_{20}$ clusters in the gas phase. Science 321, 674-676 (2008).

57. Vargas, A., Santarossa, G., Iannuzzi, M. \& Baiker, A. Fluxionality of gold nanoparticles investigated by Born-Oppenheimer molecular dynamics. Phys Rev. B: Condens. Matter Mater. Phys. 80, 1-13 (2009).

58. Austin, N., Johnson, J. K. \& Mpourmpakis, G. $\mathrm{Au}_{13}$ : $\mathrm{CO}$ adsorbs, nanoparticle responds. J. Phys. Chem. C 119, 18196-18202 (2015).

59. Becke, A. D. Density-functional exchange-energy approximation with correct asymptotic behavior. Phys. Rev. A 38, 3098-3100 (1988).

60. Perdew, J. P. Density-functional approximation for the correlation energy of the inhomogeneous electron gas. Phys. Rev. B 33, 8822-8824 (1986).

61. Weigend, F., Häser, M., Patzelt, H. \& Ahlrichs, R. RI-MP2: optimized auxiliary basis sets and demonstration of efficiency. Chem. Phys. Lett. 294, 143-152 (1998).

62. Weigend, F. \& Häser, M. RI-MP2: first derivatives and global consistency. Theor. Chem. Acc. 97, 331 (1997).

63. Feyereisen, M., Fitzgerald, G. \& Komornicki, A. Use of approximate integrals in ab initio theory. An application in MP2 energy calculations. Chem. Phys. Lett. 208, 359-363 (1993).

64. Ahlrichs, R. et al. Electronic-structure calculations on workstation computersthe program system Turbomole. Chem. Phys. Lett. 162, 165-169 (1989).

65. Tian, S. et al. Structural isomserism in gold nanoparticles revealed by X-ray crystallography. Nat. Commun. 6, 8667 (2015).

66. Uematsu, M. \& Frank, E. U. Static dielectric constant of water and steam. J. Phys. Chem. Ref. Data 9, 1291 (1980).

67. Klamt, A. \& Schuurmann, G. COSMO: a new approach to dielectric screening in solvents with explicit expressions for the screening energy and its gradient. J. Chem. Soc. Perkin Trans. 2 5, 799 (1993).

68. Fernando, A. \& Aikens, C. M. Ligand exchange mechanism on thiolate monolayer protected $\mathrm{Au}_{25}(\mathrm{SR})_{18}$ nanoclusters. J. Phys. Chem. C 119, 20179-20187 (2015).

69. Kittel, C. Introduction to solid state physics. Am. J. Phys. 35, 547 (2004).

70. Reed, A. E., Weinstock, R. B. \& Weinhold, F. Natural population analysis. J. Chem. Phys. 83, 735-746 (1985).

\section{Acknowledgements}

This material is based upon work supported by the National Science Foundation (CBET-CAREER program) under Grant No. 1652694. M.G.T. acknowledges support by 
the National Science Foundation Graduate Research Fellowship under Grant No. 1247842. The authors would like to acknowledge computational support from the Center for Simulation and Modeling (SAM) and the Extreme Science and Engineering Discovery Environment, which is supported by the NSF (ACI-1548562). The authors would also like to thank Professor Rongchao Jin from Carnegie Mellon University, for suggesting experimental structures reported in literature.

\section{Author contributions}

M.G.T. performed all the calculations. G.M. conceived the project and carried out the advising. Both authors aided in the development and writing of this manuscript.

\section{Additional information}

Supplementary Information accompanies this paper at http://www.nature.com/ naturecommunications

Competing interests: The authors declare no competing financial interests.

Reprints and permission information is available online at http://npg.nature.com/ reprintsandpermissions/

How to cite this article: Taylor, M. G. \& Mpourmpakis, G. Thermodynamic stability of ligand-protected metal nanoclusters. Nat. Commun. 8, 15988 doi: $10.1038 /$ ncomms15988 (2017).

Publisher's note: Springer Nature remains neutral with regard to jurisdictional claims in published maps and institutional affiliations.

(c) (i)

Open Access This article is licensed under a Creative Commons Attribution 4.0 International License, which permits use, sharing, adaptation, distribution and reproduction in any medium or format, as long as you give appropriate credit to the original author(s) and the source, provide a link to the Creative Commons license, and indicate if changes were made. The images or other third party material in this article are included in the article's Creative Commons license, unless indicated otherwise in a credit line to the material. If material is not included in the article's Creative Commons license and your intended use is not permitted by statutory regulation or exceeds the permitted use, you will need to obtain permission directly from the copyright holder. To view a copy of this license, visit http://creativecommons.org/ licenses/by/4.0/

(C) The Author(s) 2017 